ence scholars in family medicine to participate in the work of IOM and further their careers as future leaders in the field.

IOM Anniversary Fellows continue their main responsibilities while engaging part-time over a twoyear period in the IOM's health and science policy work. A committee appointed by the president of the IOM selects fellows based on their professional accomplishments, potential for leadership in health policy in the field of family medicine, reputation as scholars, and the relevance of their expertise to the work of the IOM.

Communications Department American Board of Family Medicine

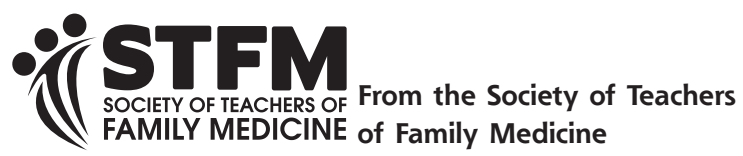

Ann Fam Med 2015;13:188-189. doi: 10.1370/afm.1775.

\section{CREATING LEADERS IN FAMILY MEDICINE}

Building leaders in family medicine is critical as our discipline embarks on the Family Medicine for America's Health Initiative. Do family medicine faculty members have the leadership skills to lead the discipline in this transformation? While we all recognize the need to be advocates for our patients, leaders in our community, and champions of our specialty, where does the training come from to do this effectively?

Too often we assume that if someone is an effective clinician, they can transition to be an effective leader. The skills required to provide comprehensive, compassionate care to patients may be important in a leader, yet alone are not sufficient. The family of family medicine shares in the responsibility to help provide this training. STFM offers many leadership training opportunities to help the discipline of family medicine grow more leaders.

\section{Behavioral Science/Family Systems Educator Fellowship}

This is a competitive, year-long fellowship experience designed for any family medicine faculty member who has responsibility for coordinating or teaching the behavioral science/family systems curriculum in their departments or residencies. Ideally suited for applicants with 5 or fewer years of experience as a faculty member, the fellowship includes a structured learning curriculum of core content and formalized mentoring that will enable participants to understand the medical culture and learn how to increase the value of integrat- ing behavioral science and family systems core principles into the practice of family medicine. All fellows also complete a mentored scholarly project.

\section{Emerging Leaders Program}

Through the Emerging Leaders program, participants learn about leadership, with an emphasis on the selfreflection and self-awareness skills required of a good leader. This program is unique because each participant is immersed into the leadership experience from the first day, with actual experience selecting talent, chairing conference calls, motivating others, and leading. Participants in the Emerging Leader program complete a project that involves leading a group to complete a task or initiative for STFM. A personal coach offers each participant advice and feedback during his/her fellowship experience and through completion of a project.

\section{Faculty for Tomorrow}

Faculty for Tomorrow is a 2-year campaign to address the shortage of family medicine faculty. The campaign will focus on strategies to expand STFM's formal faculty recruiting of residents, provide resources and training for new faculty, identify and support young family physicians with leadership potential, and ensure leaders of institutions understand the time and competencies required to be faculty.

\section{Family Medicine Congressional Conference Scholars}

Scholars will join Family Medicine Congressional Conference attendees in learning about the legislative process, current priorities for family medicine, and how they can take action and interact with legislators and health care leaders. Each scholar will also be connected to a mentor who will guide them as they prepare for the conference

\section{Leading Change Curriculum}

The Leading Change online curriculum was developed to educate and empower family medicine faculty to lead change within their institutions, larger surrounding environments, and their nation. The curriculum includes a series of 12 modules to help family medicine faculty, at all levels of their careers, plan for and implement change. Module topics include change leadership fundamentals, team building, change plan development, outcomes measurement, effective teamwork, and coaching skills.

\section{The Medical Student Educators Development Institute (MSEDI)}

The Institute is a year-long comprehensive learning experience designed to give faculty the skills to 
advance their career in medical student education. The Medical Student Educators Development Institute (MSEDI) teaches grant writing, negotiating, curriculum and exam development, student evaluation, as well as obtaining and managing resources and directing a clerkship. MSEDI participants forge enduring relationships, with both other participants as well as the Institute faculty, who support and guide them throughout their professional careers.

\section{New Faculty Scholars}

The New Faculty Scholars Award provides STFM members who are in their first 2 years as full-time faculty, and who exhibit outstanding leadership potential, the opportunity to attend the STFM Annual Spring Conference. Scholars receive exposure to discipline leaders and community-building activities that might not have occurred without the program.

\section{Other STFM Leadership Opportunities}

Regardless of where an individual is in their career path, there is a leadership role to fit varied interests and skills. Leadership opportunities include chairing a STFM special interest group, heading a task force, serving on a standing committee, or serving as a mentor, publication reviewer, fellowship faculty member, or a liaison to other organizations. There is an opportunity to fit each individual's need, interest, and time commitment.

Creating organizational culture, managing change, recognizing the systems barriers to progress and opportunities for improvement require, among others, skills in negotiation, conflict management, and strategic thinking. Only by dedicated attention to acquiring, practicing, and implementing leadership skills can we hope to develop effective leaders for family medicine.

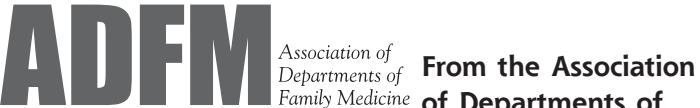 VISION, VOICE, LEADERSHIP Family Medicine}

Ann Fam Med 2015;13:189-190. doi: 10.1370/afm.1770.

\section{BUILDING RESEARCH CAPACITY IN DEPARTMENTS OF FAMILY MEDICINE: PEARLS FROM NAPCRG 2013}

One of the ongoing goals of the ADFM research committee is to assist chairs in building research capacity in family medicine departments. As a part of this effort, there were 3 separate events at the 2013 North
American Primary Care Research Group (NAPCRG) annual meeting focusing on this issue. The first event was actually 9 events-_mini-consultations" given by chairs with research experience to those working to develop research within their departments. The second was a 90-minute session called "Secrets of My Research Success," during which 3 experienced and successful family medicine researchers told their stories and answered questions from an engaged group of about 70 participants. Finally, approximately 17 Canadian and US family medicine chairs in attendance at NAPCRG conducted their customary NAPCRG joint meeting, and the focus was building research capacity. We (J.H., T.K.) had the privilege of attending all 3 sessions and would like to share the take-away lessons. These lessons are for both department chairs and aspiring researchers.

\section{General Advice}

Start with small steps, but be persistent. Don't think of research as a series of projects, but rather as a program of inquiry in which one study sets the stage for the next. Both mosaic (variety of areas of inquiry) and single theme models of research divisions can work. It depends on faculty interest and skills. Embrace critical thinking, which is essential for high quality research designs. Good researchers must embrace their critics and learn from others' experiences and suggestions.

\section{Finding a Research Question/Area of Inquiry} Be sure you answer the "so what" question first-your area of inquiry must be compelling. Good sources of research questions arise from puzzles and predicaments that motivate the researcher. But don't "jump in an intellectual box without a lid"—-be ready to jump out and reconceptualize your research if it is leading nowhere. For example, studying a condition only in the clinic setting may be too small a box. What happens to these patients in the community?

\section{Getting Experience}

Present posters as a first step, which can build confidence for publishing your findings and help you find collaborators. Or start by writing an evidence-based review. Become an expert in a content area you are passionate about, and good research questions will emerge.

\section{Training and Mentorship}

Consider a multiple mentor model—one for career guidance and others for content/methods expertise. Don't only look up for mentors-also look sideways; peers at a similar level of development can be highly effective mentors. Look outside your department 